\title{
The Mumford Form and the Polyakov Measure in String Theory
}

\author{
A. A. Beilinson ${ }^{1}$ and Yu. I. Manin ${ }^{2}$ \\ 1 State Cardiology Center, Moscow, USSR \\ 2 Steklov Mathematical Institute, Moscow, USSR
}

\begin{abstract}
An explicit formula is derived for the Mumford form on the moduli space of algebraic curves. According to the Belavin-Knizhnik theorem, this gives a formula for the Polyakov bosonic string measure.
\end{abstract}

\section{Introduction}

\section{Polyakov's String}

The quantum bosonic Polyakov string theory is defined by a path integral taken over random surfaces in the (Euclidean) $d$-dimensional space $\mathbb{R}^{d}$. The partition function of the closed string has a perturbation series expansion $Z=\sum_{g \geqq 0} Z_{g}$ :

$$
\begin{gathered}
Z_{g}=e^{\beta(2-2 g)} \int e^{-J(x, \gamma)} D x D \gamma, \\
J(x, \gamma)=\int_{N} d^{2} z \sqrt{|\gamma|} \gamma^{a b} \partial_{a} x^{\mu} \partial_{b} x^{\mu} .
\end{gathered}
$$

Here $N$ is a fixed compact oriented surface of genus $g$ (= "loop number"), $z^{a}$ are local coordinates on it, $x=\left(x^{\mu}\right)$ is a map from $N$ to $\mathbb{R}^{d}, \gamma_{a b} d z^{a} d z^{b}$ is a metric on $N$.

On the space of classical fields $(x, \gamma)$ a gauge group $C \ltimes D$ acts, leaving the classical action $J(x, \gamma)$ invariant. It is a semidirect product of the diffeomorphism group $D$ of $N$ and of the conformal group $C$ (= real-valued positive functions on $N)$. Using this action, we can reduce (1) to a finite-dimensional integral in the following way. First, the integral over $x$ 's is Gaussian, hence it equals $\left(\operatorname{det}^{\prime} \Delta_{0_{\gamma}}\right)^{-d / 2}$, where $\Delta_{0 \gamma}$ is the Laplace operator on the functions on $N$, corresponding to $\gamma, \operatorname{det}^{\prime}$ its determinant without zero modes, regularized, say, by the formula $\operatorname{det}^{\prime} \Delta_{0 \gamma}$ $=\exp \left(-\zeta_{\Delta}^{\prime}(0)\right)$. The remaining integration over the space $\operatorname{Met} N$ of $\gamma$ 's then reduces, via the Faddeev-Popov trick, to an integral over Met $N / C \ltimes D$, which is the same as the moduli space of Riemannian surfaces (or complex algebraic curves) of genus $g$. As is well known, this moduli space $M_{g}$ is a complex variety of complex dimension 0 for $g=0,1$ for $g=1,3 g-3$ for $g \geqq 2$. 
Strictly speaking, this reduction is spoiled by the conformal anomaly: the regularization breaks the conformal invariance of the quantum effective action. But the anomaly cancels in the critical dimension $d=26$ (cf. [2,3]), and one has

$$
Z_{g}=\text { const } \int_{M_{g}} d v \operatorname{det}^{\prime} \Delta_{2 \hat{\gamma}}\left(\operatorname{det}^{\prime} \Delta_{0 \hat{\gamma}}\right)^{-13}, \quad g \geqq 2,
$$

where $d v$ is the Petersson-Weil measure on the moduli space, $\Delta_{2 \hat{\gamma}}$ is a Laplace operator on quadratic differentials, $\hat{\gamma}$ is a metric of constant curvature -1 in a given conformal class (for $g=1$ the formula differs slightly).

\section{Results}

The main result of this paper (Theorem 6, Sect. 1) is an explicit formula for the Polyakov measure $d \pi_{g}$ on $M_{g}$, appearing in the right-hand side of (2). Here "explicit" means "written in terms of the complex geometry of the surface itself" and not in terms of it spectral invariants. Note that certain explicit formulae for the Laplace operator determinants were given by Ray and Singer [20]. In several papers, including $[5,21]$ they were applied to the string measure case. In this approach, $\log \operatorname{det}^{\prime} \Delta$ is transformed into a sum over lengths of closed geodesics by means of the Selberg trace formula.

The formula of our paper looks very differently and is of a different nature since it utilizes the holomorphic invariants and not the metric ones.

One may consider this formula as a generalization to the genus $g \geqq 2$ case of the well known genus 1 result:

$$
\begin{aligned}
& Z_{1}=\int_{M_{1}^{\prime}} \frac{i}{2} d \tau \wedge d \bar{\tau} \frac{1}{(\operatorname{Im} \tau)^{14}|\Delta(\tau)|^{2}}, \\
& \Delta(\tau)=e^{2 \pi i \tau} \prod_{n=1}^{\infty}\left(1-e^{2 \pi i n \tau}\right)^{24}, \\
& M_{1}^{\prime}=\left\{\tau|| \tau|\geqq 1,| \operatorname{Re} \tau \mid \leqq \frac{1}{2}, \operatorname{Im} \tau>0\right\} .
\end{aligned}
$$

Observe that (3) may be expressed in terms of the theta function,

$$
\theta(z, \tau)=\sum_{n=-\infty}^{\infty} e^{2 \pi i n z+\pi i n^{2} \tau},
$$

since

$$
2^{8} \Delta(\tau)=e^{2 \pi i \tau}(\theta(0, \tau) \theta(1 / 2, \tau) \theta(\tau / 2, \tau))^{8} .
$$

\section{How to Compute Polyakov's Measure}

The computation presented here is based on the results of Mumford [8] and of Belavin and Knizhnik [7]. Here is a brief summary of their work.

The moduli space $M_{g}$ is a complex algebraic variety. It has no convenient global coordinates. However, holomorphic 1-forms on $M_{g}$ admit the following nice description: they correspond to the quadratic differentials on the complex curves, parametrized by the points of $M_{g}$. Let $w_{1}, \ldots, w_{3 g-3}($ here $g \geqq 2$ ) be a basis of such differentials (depending holomorphically on parameters from $M_{g}$ ), and let 
$\omega_{1}, \ldots, \omega_{g}$ be a basis of differentials of the first kind. Mumford [8] shows, that there exists a unique, up to a constant multiple, holomorphic function $F$ (depending on the choice of $\left.w_{i}, \omega_{j}\right)$, such that

$$
\mu_{g}=F \cdot \frac{w_{1} \wedge \ldots \wedge w_{3 g-3}}{\left(\omega_{1} \wedge \ldots \wedge \omega_{g}\right)^{13}}
$$

is a global section of the appropriate line bundle on $M_{g}$. This section is meromorphic at infinity (i.e., it is algebraic) and has a pole of order two; moreover, it has no zeroes on $M_{g}$. (Actually these properties are automatic for $g \geqq 3$, and for $g=2$ they should be imposed additionally.)

We shall call $\mu_{g}$ a Mumford form.

Comparing this with (2), one is lead to believe that "13" in Mumford's formula coincides with the half of the critical dimension. This was suggested by Yu. I. Manin and supported by certain evidence from the operator quantization approach.

In a remarkable paper [7] Belavin and Knizhnik pushed this much further. Namely, they proved that the Polyakov measure coincides with the modulus squared of the Mumford form. More precisely, let $W_{i}$ be a 1 -form on $M_{g}$, corresponding to $w_{i}$, then one has

$$
d \pi_{g}=\mathrm{const}|F|^{2} \cdot(-i)^{g} \frac{W_{1} \wedge \bar{W}_{1} \ldots W_{3 g-3} \wedge \bar{W}_{3 g-3}}{\left|\operatorname{det} \int \omega_{i} \wedge \bar{\omega}_{j}\right|^{13}} .
$$

Here bar means complex conjugate, and the integral is taken over the Riemann surface on which $\omega_{i}$ 's are defined.

Hence to compute $d \pi_{g}$ it suffices to describe explicitly the Mumford form, a priori defined only by the implicit global conditions. This is the task we concentrate upon in what follows.

Note that in [14] $d \pi_{g}$ already was calculated in this way. This was done with the help of Faltings' paper [9], which developed in turn the ideas of Arakelov, namely the Noether theorem for arithmetic surfaces. The formula presented below is shorter than the one in [14]. We hope also that its derivation clarifies somewhat the arguments of Faltings.

The expression for the curvature form of the determinant of the Laplace operator on $j$-differentials, found by Belavin and Knizhnik and directly implying (5), appears to be a particular case of the similar formula for the determinants of Dirac operators on arbitrary compact manifolds, due to Bismut and Freed [17] (this was pointed out in [18]). The Bismut-Freed result (generalizing the earlier theorem of Quillen) in the context of complex Hermitian geometry may be viewed as an exact Riemann-Roch-Grothendieck formula for $c_{1}$, valid on the level of forms, and not on the cohomology level only. A similar formula for higher $c_{i}$ remains to be found. We are sure it will help to understand both anomalies in physics and arithmetic geometry, in the spirit advocated in [19].

\section{Superstrings}

The Polyakov string has a fermion analog, described in $[2,4]$. The critical dimension for the superstring is $d=10$. A computation of an analogue of $d \pi_{g}$ on the 
corresponding moduli superspaces was done in [6] by means of a supervariant of the Selberg trace formula. However, the relation with the right Polyakov measure seems not quite clear, due to two circumstances. a. The superversion of the BelavinKnizhnik theorem is not available at the moment. b. One does not know how to sum up a Mumford superform over different spin structures before taking the modulus squared. We discuss this question briefly in Sect. 3.2.

\section{The Contents of the Paper}

Our basic result is, after some preliminaries, stated in Sect.1.6. Its proof is presented in Sect. 2. The third section contains some remarks and complements.

Finally, the appendix deals with the following problem. Mumford forms $\mu_{g}$ are defined a priori only modulo multiplication by a constant, depending on the genus. We shall explain how one can normalize all $\mu_{g}$ 's canonically and simultaneously. Conjecturally, one should have $e^{\beta(2-2 g)}\left|\mu_{g}\right|^{2}=d \pi_{g}$ with this normalization.

\section{Notation and Statement of Results}

\section{Calculus on Riemann Surface}

Let $X$ be a compact complex Riemann surface of genus $g \geqq 1$, or, equivalently, a smooth projective algebraic curve over $\mathbb{C}$. Marking of $X$ is a choice of a symplectic basis $\left(a_{1}, \ldots, a_{g} ; b_{1}, \ldots, b_{g}\right)$ in $H_{1}(X, \mathbb{Z})$. This means that $\left(a_{i}, a_{j}\right)=\left(b_{i}, b_{j}\right)=0,\left(a_{i}, b_{j}\right)$ $=\delta_{i j}$. One can construct such a basis making a classical system of cuts, which turns $X$ into a polygon with pairwise identified sides (see e.g. [12]).

The space of holomorphic 1-forms (or differentials of the first kind) on $X$ has the Riemann basis $\omega_{1}, \ldots, \omega_{g}$ uniquely determined by the conditions $\int_{a_{i}} \omega_{j}=\delta_{i j}$, $i, j=1, \ldots, g$. Put $\int_{b_{i}} \omega_{j}=\tau_{i j}, \tau=\left(\tau_{i j}\right)$. One has $\tau_{i j}=\tau_{j i}$, Im $\tau>0$, hence $\tau$ lies in the Siegel upper halfspace $H_{g}$. The curve $X$ together with its marking can be reconstructed from $\tau$ up to unique isomorphism.

Let $T \subset \mathbb{C}^{g}$ be a lattice, generated by columns of $\tau$ and $\mathbb{Z}^{g}$. The Jacobian of $X$ is the complex torus $J=\mathbb{C}^{g} / T$. For each $P_{0} \in X, z_{0} \in J$ there is a standard embedding $\psi: X \rightarrow J$, mapping $P_{0}$ to $z_{0}$, defined by the formula $\psi(P)=\left(z_{0}+\int_{P_{0}}^{P} \omega\right) \bmod T$, $\omega=\left(\omega_{i}\right)$. If $z_{i}$ is the $i^{\text {th }}$ coordinate on $\mathbb{C}^{g}$, the $T$-invariant form $d z_{i}$ on $\mathbb{C}^{g}$ may be viewed as a holomorphic (translation invariant) form on $J$. Any standard embedding $\psi: X \rightarrow J$ induces the same isomorphism between the space of such forms on $J$ and the space of the differentials of the first kind on $X: \psi^{*}\left(d z_{i}\right)=\omega_{i}$.

The Jacobian $J$ classifies the divisor classes of degree zero on $X$, or, equivalently, the isomorphism classes of invertible sheaves $L$ on $X$ with $c_{1}(L)=0$. Namely, a sheaf $O_{X}\left(\sum a_{i} P_{i}\right)$ corresponds to a point $\sum a_{i} \psi\left(P_{i}\right)$, where $\psi$ is any standard embedding.

\section{Theta Function}

The function $\theta: \mathbb{C}^{g} \times H_{g} \rightarrow \mathbb{C}$ is defined by the classical series

$$
\theta(z, \tau)=\sum_{m \in \mathbb{Z}^{g}} \exp 2 \pi i\left(m^{t} z+1 / 2 m^{t} \tau m\right) \text {. }
$$


It has a kind of periodic behavior:

$$
\begin{aligned}
& \theta(z+m, \tau)=\theta(z, \tau), \\
& \theta(z+\tau n, \tau)=\theta(z, \tau) \exp 2 \pi i\left(-n^{t} z-1 / 2 m^{t} \tau n\right) .
\end{aligned}
$$

In particular, the zero divisor of $\theta(z, \tau)$ (as a function of $z$; $\tau$ being fixed) is $T$-invariant and hence comes from a divisor $\Theta$ on $J$.

For $\alpha=\varepsilon+\tau \delta \in \mathbb{C}^{g}$, put

$$
\theta[\alpha](z, \tau)=\exp 2 \pi i\left(\delta^{t}(z+\varepsilon)+1 / 2 \delta^{t} \tau \delta\right) \theta(z+\varepsilon+\tau \delta, \tau) .
$$

If $\varepsilon, \delta \in 1 / 2 \mathbb{Z}^{g}$, then the class $\alpha \bmod T \in J$ is called theta characteristics. The parity of such $\alpha$ is $4 \varepsilon \delta \bmod 2$. The function $\theta[\alpha](z, \tau)$ is even or odd with respect to $z$ according to the parity of $\alpha$. The theta characteristics are the second order points on $J$. Their total number is $4^{g}$, of which there are $2^{g}\left(2^{g}-1\right)$ even ones and $2^{g}\left(2^{g}+1\right)$ odd ones.

\section{Moduli of Curves and Moduli of Abelian Varieties}

The complex tori, corresponding to the points $\tau \in H_{g}$, are algebraic. They are called abelian varieties. The Siegel halfspace $H_{g}$ of dimension $g(g+1) / 2$ parametrizes the pairs $\left(A,\left(a_{i}, b_{j}\right)\right)$, where $A$ is a principally polarized abelian variety and $\left(a_{i}, b_{j}\right)$ is a symplectic basis in $H_{1}(A, \mathbb{Z})$. Those pairs that come from (Jacobians of) curves from a closed analytic subvariety $N_{g} \subset H_{g}$ of dimension 1 for $g=1,3 g-3$ for $g \geqq 2$. Hence for $g \geqq 4$ one has $\operatorname{dim} N_{g}<\operatorname{dim} H_{g}$.

To the same curve there corresponds many points of $N_{g}$, since we can change the marking. Such a change transforms $\tau$ by an element

$$
\left(\begin{array}{ll}
A & B \\
C & D
\end{array}\right) \in \mathrm{Sp}(2 g, \mathbb{Z}): \tau \mapsto(A \tau+B)(C \tau+D)^{-1} .
$$

This action of $\operatorname{Sp}(2 g, \mathbb{Z})$ on $H_{g}, N_{g}$ is discrete. The space $\operatorname{Sp}(2 g, \mathbb{Z}) \backslash N_{g}=M_{g}$ is called the (coarse) moduli space of curves of genus $g$, and $\operatorname{Sp}(2 g, \mathbb{Z}) \backslash H_{g}=A_{g}$ is the (coarse) moduli space of (principally polarized) abelian varieties.

The functions $\tau_{i j}, i \geqq j$, are global holomorphic coordinates on $H_{g}$. Locally they are coordinates at any smooth point of $A_{g}$ (or a covering space of $A_{g}$ ). And, although the the functions $\tau_{i j}$ being restricted to $N_{g}$, become dependent (for $g \geqq 4$ ), at any point of $N_{g}$ corresponding to a nonhyperelliptic curve $X_{x}$, one may choose $3 g-3$ of $\tau_{i j}$ 's that form local coordinates at $x$ on $N_{g}$. To be more precise, let $k$ be the Kodaira-Spencer map identifying the quadratic differentials on $X_{x}$ with the fiber of $\Omega^{1} N_{g}$ at $x$. Then one has $k\left(\omega_{a} \omega_{b}\right)=(2 \pi i)^{-1} d \tau_{a b}$. Below we shall describe the Mumford form in terms of $\left(\omega_{a}\right),\left(\omega_{a} \omega_{b}\right)$. Processing it into the Polyakov measure one should replace $\omega_{a} \omega_{b}$ by $\left.(2 \pi i)^{-1} d \tau_{a b}\right|_{N_{g}}$.

\section{Theta Characteristics and Differentials of Half-Integral Weight}

For any integer $d$ denote by $J_{d}$ the variety, parametrizing the isomorphism classes of the invertible sheaves of degree $d$ on $X$. This is a principal homogeneous space over $J$. For almost all sheaves $L$ of degree $g-1$ one has $h^{0}(L)=h^{1}(L)=0$. More precisely, the sheaves with $h^{0}(L)>0$ form a divisor $\Theta_{g-1} \subset J_{g-1}$. According to the 
Riemann theorem, there exists a unique isomorphism $J \rightarrow J_{g-1}$, compatible with $J$-action, which maps $\Theta$ to $\Theta_{g-1}$. It identifies $0 \in J$ with the Riemann point $\Delta \in J_{g-1}$. One has $2 \Delta=$ class of $\Omega^{1}$ in $J_{2 g-2}$, where $\Omega^{1}$ is the sheaf of 1 -forms on $X$ (see $[12,15]$ ). Note that $\Delta$ depends on the marking of $X$. In this way the set of theta characteristics transforms into the set of square roots of $\Omega^{1}$. Therefore below we shall indiscriminately call "theta characteristics" a point in $J$, or in $J_{g-1}$ (a half of $\Omega^{1}$ ), or else a sheaf $L_{\alpha}$ together with an isomorphism $L_{\alpha}^{\otimes 2} \underset{\sim}{\rightarrow} \Omega^{1}$.

For any curve $X$ there exists such an odd $\alpha$ (the "typical" odd one), that $h^{0}\left(L_{\alpha}\right)=1$. We choose one and fix it. We put $\Omega^{1 / 2}=L_{\alpha}$ and $\Omega^{i}=L_{\alpha}^{\otimes 2 i}$ for $i \in 1 / 2 \mathbb{Z}$.

\section{Distinguished Bases}

Using $\Omega^{1 / 2}$ we shall define now certain bases in the spaces of differentials and quadratic differentials on $X$. We assume that $g \geqq 2$ and that $X$ is not hyperelliptic, if $g \geqq 3$.

a) The basis $\left(\varphi_{0}, \varphi_{1}, \ldots, \varphi_{g-1}\right)$ in $\Omega^{1}(X)$. Put

$$
\varphi_{0}=\sum_{j=1}^{g}\left(\partial / \partial z_{j} \theta[\alpha]\right)(0, \tau) \omega_{j}
$$

This differential has double zeroes on $X$. Assume that $\operatorname{div} \varphi_{0}=\sum_{i=1}^{g-1} 2 P_{i}$ with pairwise different $P_{i}$ (this is true for an open dense subset in $N_{g}$ ). We have $\varphi_{0}=v_{\alpha}^{2}$, where $v_{\alpha}$ is a certain $1 / 2$-differential.

Choose local parameters $t_{j}$ at $P_{j}$ in such a way that $\varphi_{0}=t_{j}^{2} d t_{j}$. Clearly, $t_{j}(P)$ $=\left(3 \int_{P_{J}}^{P} \varphi_{0}\right)^{1 / 3}$, so that $t_{j}$ are defined up to multiplication by $\sqrt[3]{1}$

Now the conditions

$$
\varphi_{j}=\left(\delta_{j k}+a_{j k} t_{k}\right) d t_{k}+O\left(t_{k}^{3} d t_{k}\right) \text { near } P_{k} ; j, k=1, \ldots, g-1
$$

define the differentials $\varphi_{j}$ uniquely. Put moreover

Clearly, $(\varphi)^{t}=B(\omega)^{t}$.

$$
B=\left(B_{i j}\right)=\left(\int_{a_{j}} \varphi_{i-1}\right), \quad i, j=1, \ldots, g
$$

b) The basis $\left(w_{1}, \ldots, w_{3 g-3}\right)$ in $\Omega^{2}(X)$. For $g=2$ or for non-hyperelliptic $X$ $(g \geqq 3)$ put

$$
\begin{aligned}
&\left(w_{1}, \ldots, w_{3 g-3}\right)=\left(\varphi_{0}^{2}, \varphi_{0} \varphi_{1}, \ldots, \varphi_{0} \varphi_{g-1} ; \varphi_{1}^{2}, \ldots, \varphi_{g-1}^{2}\right. \\
&\left.a_{1, g-1}^{-1} \varphi_{1} \varphi_{g-1}, \ldots, a_{g-2, g-1}^{-1} \varphi_{g-2} \varphi_{g-1}\right)
\end{aligned}
$$

The last group of these differentials is defined on an open dense subset of $N_{g}$, where all $a_{i, g-1}$ are invertible. Off this subset one should change this choice as explained in Sect. 2. The only important thing is that

$$
w_{2 g-1+j}=\delta_{j k} t_{k}\left(d t_{k}\right)^{2}+O\left(t_{k}^{2}\left(d t_{k}\right)^{2}\right) \text { near } P_{k}, \quad j, k=1, \ldots, g-2,
$$

and also a possibility to explicitly present $w_{i}$ as bilinear combinations of $\omega_{a} \omega_{b}$ for computation of the map $k$.

We can now write down formulae for $\mu_{g}$ and $d \pi_{g}$. 
6.

Theorem. a) For $g \geqq 2$ the Mumford form is

$$
\mu_{g}=\operatorname{const}(\operatorname{det} B)^{4} \frac{w_{1} \wedge \ldots \wedge w_{3 g-3}}{\left(\varphi_{0} \wedge \ldots \wedge \varphi_{g}\right)^{13}}
$$

b) In the same conditions the Polyakov measure is

$$
d \pi_{g}=\mathrm{const}|\operatorname{det} B|^{-18}(\operatorname{det} \operatorname{Im} \tau)^{-13} W_{1} \wedge \bar{W}_{1} \wedge \ldots \wedge W_{3 g-3} \wedge \bar{W}_{3 g-3},
$$

where $W_{i}=k\left(w_{i}\right), k\left(\omega_{a} \omega_{b}\right)=(2 \pi i)^{-1} d \tau_{a b}$.

\section{Proofs}

1. $\operatorname{det} R \pi_{*}$ formalism

Let $\pi: X \rightarrow S$ be an algebraic family of projective varieties $X_{s}$, parametrized by points $s \in S$, or, as an algebraic geometer would say, just a projective flat morphism. Consider an (algebraic coherent) sheaf $L$ on $X$, flat over $S$; roughly speaking, this is a family of sheaves $L_{s}$ on the fibers of $\pi$. If for a certain $i$ the dimensions of the cohomology groups $H^{i}\left(X_{s}, L_{s}\right)$ do not depend on $s$, then they are fibers of the higher direct image sheaf $R^{i} \pi_{*} L$, and this sheaf is locally free. If this holds for all $i$, we may define a "multiplicative Euler characteristic"

$$
d(L):=\bigotimes_{i}\left(\operatorname{det} R^{i} \pi_{*} L\right)^{(-1)^{i}}:=\operatorname{det} R \pi_{*} L,
$$

which is an invertible sheaf on $S$.

Knudsen and Mumford in [10] have shown how to define $d(L)$ with nice properties for any $L$ flat over $S$, without assuming that $\operatorname{dim} H^{i}\left(X_{s}, L_{s}\right)$ does not jump. To be more precise, they proved the following result.

\section{2.}

Proposition. For any family of projective varieties $\pi: X \rightarrow S$, any flat sheaf $L$ on $X$ and any isomorphism of sheaves $f: L \rightarrow L^{\prime}$ one can construct an invertible sheaf $d(L)$ on $S$ and an isomorphism $d(f): d(L) \rightarrow d\left(L^{\prime}\right)$ in such a way that $d$ becomes a functor with the following properties. (Below equalities mean canonical isomorphisms.)

a) $d(L)=\operatorname{det} R \pi_{*} L$, if all $R^{i} \pi_{*} L$ are locally free.

b) $d(L)$ is compatible with base change (= change of parameter space $S$ ).

c) Let $E=\left(E^{i}, d^{i}\right)$ be a finite complex of locally free sheaves on $S$, whose cohomology is $R^{i} \pi_{*} L$ universally (i.e. after any base change). (See [13] for an explanation of this Grothendieck's construction). Then $d(L)=\bigotimes_{i}\left(\operatorname{det} E^{i}\right)^{(-1)^{i}}$.

d) For any exact triple $0 \rightarrow L^{\prime} \stackrel{i}{\longrightarrow} L \stackrel{j}{\longrightarrow} L^{\prime \prime} \rightarrow 0$ one has a canonical isomorphism $d(L)=d\left(L^{\prime}\right) \otimes d\left(L^{\prime \prime}\right)$, compatible with exact triples of exact triples and base change.

Note that if $a: L \rightarrow L$ is multiplication by $a \in \mathbb{C}^{*}$, then $d(a)$ is multiplication by $a^{\chi(L)}$, where $\chi(L)=\sum(-1)^{i} r k R^{i} \pi_{*} L$. This explains the name "multiplicative Euler characteristics." 
We refer to [10] for further details. In concrete computations below we shall not use much more than the following particular case. Assume that $R^{i} \pi_{*} L=0$ for $i \geqq 1$ and let $l=\left(l_{1}, \ldots, l_{a}\right)$ denote some free generators of $R^{0} \pi_{*} L$. Then $d(L)$ is freely generated by

$$
d(l)=l_{1} \wedge \ldots \wedge l_{a} \in \operatorname{det} R^{0} \pi_{*} L
$$

If the same holds for the terms $L, L^{\prime}, L^{\prime \prime}$ of an exact triple, as in d) above, and if the bases are chosen in such a way that

$$
l^{\prime}=\left(l_{1}^{\prime}, \ldots, l_{a}^{\prime}\right), \quad l=\left(i\left(l_{1}^{\prime}, \ldots, l_{a}^{\prime}\right) ; l_{a+1}, \ldots, l_{b}\right), \quad l^{\prime \prime}=j\left(l_{a+1}, \ldots, l_{b}\right),
$$

then, under the canonical isomorphism $d(L)=d\left(L^{\prime}\right) \otimes d\left(L^{\prime \prime}\right)$ we have $d(l)$ $=d\left(l^{\prime}\right) \otimes d\left(l^{\prime \prime}\right)$.

\section{The Sheaves $\lambda_{i}$}

Now let $\pi: X \rightarrow S$ be a flat family of smooth projective curves, $g=$ genus, $\Omega=\Omega^{1} X / S$ the sheaf of relative 1 -forms. Put $\lambda_{i}=d\left(\Omega^{\otimes i}\right)$. We have $\lambda_{i}=\lambda_{1-i}$ in a canonical way [since, by Serre's duality, $d(L)=d\left(L^{-1} \otimes \Omega\right)$ for any invertible $L$ ].

The sheaves $\lambda_{i}$ play a crucial role in the Polyakov string measure theory due to the following facts.

a) Mumford's theorem [8]: there exists a universal isomorphism $\lambda_{i+1}=\lambda_{1}^{6 i^{2}+6 i+1}$.

This means that we have such an isomorphism for any family of curves, and we can normalize them in a way compatible with base changes. Such a universal isomorphism is unique, up to multiplication by a constant, depending on $g$ only, for $g \geqq 3$ (for $g=2$ the uniqueness also holds if certain assumptions on the behavior at infinity are added).

The uniqueness for $g \geqq 3$ follows from the fact that every holomorphic function on $M_{g}$ is constant. To see this, consider the closure of $M_{g}$ in the Satake compactification of $A_{g}$. A holomorphic function can be extended from $M_{g}$ to this closure by Hartogs' theorem, since at infinity lies a subset of codimension $\geqq 2$. But the closure is compact, hence a holomorphic function on it is constant.

In the appendix we describe a canonical normalization for all $\mathrm{g}$.

b) Theorem on modular families: if $\pi: X \rightarrow S$ is a locally universal family, then the Kodaira-Spencer map $R^{0} \pi_{*} \Omega^{\otimes 2} X / S \rightarrow \Omega^{1} S$ is an isomorphism. Passing to the determinants we get $\lambda_{2}=\Omega^{3 g-3} S$.

The combination of a) for $i=1$ and b) gives us a universal section $\mu_{g} \in \Omega^{3 g-3} S \otimes \lambda_{1}^{-13}$, called earlier the Mumford form.

c) The Belavin-Knizhnik theorem: Polyakov's measure equals const $\left|\mu_{g}\right|^{2}$ in the sense explained in the Introduction.

Below we shall reprove the Mumford theorem by a method that does not use the Riemann-Roch-Grothendieck global type arguments, and allows us to trace out the behaviour of convenient generators in $\lambda_{i}$.

Essentially this type of argument can be found in the Faltings paper [9].

Before proceeding further we need one more algebraic-geometrical construction. 


\section{The Sheaves $\langle L, M\rangle$}

Let $X$ be a smooth projective curve; $f, g$ meromorphic functions on it with disjoint divisors. Put

$$
\langle f, g\rangle=f(\operatorname{div} g)=\prod_{x \in X} f(x)^{v_{x}(g)},
$$

where $v_{x}(g)$ is the order of $g$ at a point $x$. According to A. Weil, $\langle f, g\rangle=\langle g, f\rangle$ : this generalizes the classical symmetry of the projective cross-ratio [look at the case $\left.X=P^{1}, f=(z-a)(z-b)^{-1}, g=(z-b)(z-c)^{-1}\right]$.

Assume now, that $L, M$ are invertible sheaves on $X$, and $s, t$ are their meromorphic sections with disjoint divisors. We can still define $\langle s, t\rangle=\prod_{x} s(x)^{v_{x}(t)}$ as an element of one dimensional vector space $\langle L, M\rangle_{s, t}=\bigotimes_{x} L_{x}^{\otimes v_{x}(t)}$, instead of the base field of complex numbers. But it appears, essentially due to the Weil symmetry, that these vector spaces admit a lot of canonical identifications. In particular, for a different couple of sections $s^{\prime}, t^{\prime}$ of $L, M$ one has a canonical isomorphism $\langle L, M\rangle_{s, t}=\langle L, M\rangle_{s^{\prime}, t^{\prime}}$. Hence we may omit the index $s, t$ and deal with $\langle L, M\rangle$ depending on $L, M$ only. If $X, L, M$ depend on parameters $S$, then $L, M$ form a line bundle or an invertible sheaf on $S$.

This formalism was developed by Deligne in [11]; here is a brief summary.

5.

Proposition. Let $\pi: X \rightarrow S$ be a flat family of smooth projective curves. Then for each pair of invertible sheaves $L, M$ on $X$ and each pair of isomorphisms $\varphi: L \rightarrow L^{\prime}$, $\psi: M \rightarrow M^{\prime}$, one can construct an invertible sheaf $\langle L, M\rangle$ on $S$ and an isomorphism $\langle\varphi, \psi\rangle:\langle L, M\rangle \rightarrow\left\langle L^{\prime}, M^{\prime}\right\rangle$ with the following properties.

a) This construction is a bimultiplicative symmetric bifunctor, i.e. there are natural identifications

$$
\begin{gathered}
\left\langle L, O_{X}\right\rangle=O_{S}, \quad\left\langle L_{1} \otimes L_{2}, M\right\rangle=\left\langle L_{1}, M\right\rangle \otimes\left\langle L_{2}, M\right\rangle, \\
\langle L, M\rangle=\langle M, L\rangle, \quad\left\langle L, M^{-1}\right\rangle=\langle L, M\rangle^{-1} .
\end{gathered}
$$

b) For each pair of meromorphic sections s of $L, t$ of $M$ with disjoint divisors flat over $S$, an invertible section $\langle s, t\rangle$ of $\langle L, M\rangle$ can be defined in such a way that with respect to the identifications in a) we have

$$
\begin{gathered}
\langle s, 1\rangle=1, \quad\left\langle s_{1} \otimes s_{2}, t\right\rangle=\left\langle s_{1}, t\right\rangle \otimes\left\langle s_{2}, t\right\rangle, \\
\langle s, t\rangle=(-1)^{\operatorname{deg} L \operatorname{deg} M}\langle t, s\rangle, \quad\left\langle s, t^{-1}\right\rangle=\langle s, t\rangle^{-1} .
\end{gathered}
$$

Moreover, $\langle s, f\rangle=f(\operatorname{div} s)$, if $f$ is a meromorphic function.

c) Let $M=0_{X}(D)$, where $D$ is a relative positive divisor with structure sheaf $0_{D}$, locally free over $S$. Then

$$
\begin{aligned}
\left\langle L, 0_{X}(D)\right\rangle & =\operatorname{det}_{0_{S}}\left(L \otimes 0_{D}\right) \otimes\left(\operatorname{det}_{0_{S}} 0_{D}\right)^{-1}=d\left(L \otimes 0_{D}\right) \otimes d\left(0_{D}\right)^{-1}, \\
\left\langle s, t_{D}\right\rangle= & \text { determinant of the } 0_{s} \text {-morphism } 0_{D} \rightarrow L \otimes 0_{D}, \\
& \text { sending } 1 \text { to } s \otimes 1 .
\end{aligned}
$$

Here $t_{D}$ is a canonical section of $0_{X}(D)$, equation of $D$. 
This data is compatible with arbitrary base change and is determined in a unique way, up to a unique isomorphism. result:

A relation between $d(L)$ and $\langle L, M\rangle$ is established by following Deligne's

6.

Lemma. There is a canonical isomorphism

$$
\langle L, M\rangle=d(L \otimes M) \otimes d\left(0_{X}\right) \otimes d(L)^{-1} \otimes d(M)^{-1} .
$$

The essence of this lemma is that the functor $d$ behaves in a (non-homogeneous) quadratic way with respect to the tensor product, and $\langle$,$\rangle is the associated$ bimultiplicative functor.

For our purposes we need an explicit construction of the last isomorphism. Take $M=0_{X}(-D)$. Then the exact triples

$$
\begin{gathered}
0 \rightarrow M \stackrel{t_{D}}{\longrightarrow} 0_{X} \rightarrow 0_{D} \rightarrow 0, \\
0 \rightarrow L \otimes M \rightarrow L \rightarrow L \otimes 0_{D} \rightarrow 0
\end{gathered}
$$

together with Proposition $2 \mathrm{~d}$ give

$$
\begin{gathered}
d\left(0_{D}\right)=d\left(0_{X}\right) \otimes d(M)^{-1}, \\
d\left(L \otimes 0_{D}\right)=d(L) \otimes d(L \otimes M)^{-1} .
\end{gathered}
$$

Hence, by Proposition 5c we get

$$
\begin{aligned}
\langle L, M\rangle & =\left\langle L, M^{-1}\right\rangle^{-1}=d\left(0_{D}\right) \otimes d\left(L \otimes 0_{D}\right)^{-1} \\
& =d(L \otimes M) \otimes d\left(0_{X}\right) \otimes d(L)^{-1} \otimes d(M)^{-1} .
\end{aligned}
$$

\section{Proof of Mumford's Theorem}

We shall proceed in three steps.

a)

$$
\lambda_{i+1}=\lambda_{1}\langle\Omega, \Omega\rangle^{i(i+1) / 2} .
$$

In fact, according to Lemma 6 and using $d\left(0_{X}\right)=\lambda_{1}$, we have

$$
\langle\Omega, \Omega\rangle^{i}=\left\langle\Omega^{i}, \Omega\right\rangle=d\left(\Omega^{i+1}\right) d\left(0_{X}\right) d\left(\Omega^{i}\right)^{-1} d(\Omega)^{-1}=\lambda_{i+1} \lambda_{i}^{-1} .
$$

Now induction by $i$ shows what we want. (From now on we sometimes omit $\otimes$ in notation.)

b) Assume that on the family $\pi: X \rightarrow S$ a relative theta characteristic is given, utilizing which we define $\lambda_{i}=d\left(\Omega^{1 / 2 \otimes 2 i}\right)$ for half-integral $i$ 's. Then

$$
\langle\Omega, \Omega\rangle=\lambda_{1}^{8} \lambda_{1 / 2}^{-8} \text {. }
$$

In fact,

$$
\langle\Omega, \Omega\rangle=\left\langle\Omega^{1 / 2}, \Omega^{1 / 2}\right\rangle^{4}
$$

and, by Lemma 6 ,

$$
\left\langle\Omega^{1 / 2}, \Omega^{1 / 2}\right\rangle=d(\Omega) d\left(0_{X}\right) d\left(\Omega^{1 / 2}\right)^{-2}=\lambda_{1}^{2} \lambda_{1 / 2}^{-2} .
$$


c) Under the same assumptions, $\lambda_{1}^{4} \lambda_{1 / 2}^{8}=0_{s}$. Unfortunately, we could not find a proof local by $S$ and are bound to reproduce a global and analytic argument due to Faltings. ${ }^{1}$ It consists in a reinterpretation of $\lambda_{1}$ and $\lambda_{1 / 2}$ in terms of Jacobian and in the subsequent proof of the corresponding fact for a universal family of all abelian varieties.

To do the first reduction, denote by $\Theta_{g-1} \subset J_{g-1}$ the relative theta divisor of the family and let $\alpha: S \rightarrow J_{g-1}$ be the relative theta characteristics, corresponding to $\Omega^{1 / 2}$. Finally, let $e: S \rightarrow J$ be the identity section. Then

$$
\lambda_{1 / 2}=\alpha^{*} 0\left(-\Theta_{g-1}\right), \quad \lambda_{1}=e^{*}\left(\Omega^{g} J / S\right) .
$$

For the second step recall a classical result on the behaviour of $\theta(z, \tau)$ under the simultaneous action of $\operatorname{Sp}(2 g, \mathbb{Z})$ upon $z$ and $\tau$. The group $\operatorname{Sp}(2 g, \mathbb{Z})$ consists of integral matrices $\left(\begin{array}{ll}A & B \\ C & D\end{array}\right)$ conserving the form $a\left(x_{1}, x_{2} ; y_{1}, y_{2}\right)=x_{1}^{t} y_{2}-x_{2}^{t} y_{1}$; $x_{i}, y_{j} \in \mathbb{Z}^{g}$. Let $\Gamma_{1,2}$ be the subgroup, conserving also $x_{1}^{t} x_{2} \bmod 2$ (this means that the diagonal elements of $A^{t} C, B^{t} D$ are even).

$$
\begin{aligned}
& \text { Then for all } T=\left(\begin{array}{ll}
A & B \\
C & D
\end{array}\right) \in \Gamma_{1,2} \text { one has } \\
& \qquad \begin{aligned}
\theta\left(\left((C \tau+D)^{t}\right)^{-1} z,(A \tau+B)(C \tau+D)^{-1}\right) \\
\quad=\zeta \operatorname{det}(C \tau+D)^{1 / 2} \exp \left(i z^{t}(C \tau+D)^{-1} C z\right) \theta(z, \tau),
\end{aligned}
\end{aligned}
$$

where $\zeta$ is a $8^{\text {th }}$ root of unity, depending on $T$.

The proof, given in [12], consists of two steps. First, one checks this relation for a simple generator system of $\Gamma_{1,2}$; to do this, one needs only periodicity and Poisson formula. Second, one establishes a group property of the transformation formula.

Namely, the transformation formula is equivalent to the $\Gamma_{1,2}$-invariance of certain expressions involving theta constants, in particular

$$
\left.\exp \left(2 \pi i n^{t} \tau n\right) \theta^{8}(z-1 / 2(m+\tau n), \tau)\left(d z_{1} \wedge \ldots \wedge d z_{g}\right)^{4}\right|_{z=0} .
$$

This invariance can be restated in geometric terms.

Let $\tau$ vary in the Siegel half-space $H_{g}$. Instead of $A_{g}=\operatorname{Sp}(2 g, \mathbb{Z}) \backslash H_{g}$ consider a space $A_{g}^{\prime}$, parametrizing pairs (abelian variety, degree one symmetric ample line bundle). Every such pair essentially is $\left(A, O\left(-\Theta_{\alpha}\right)\right)$, where $\alpha$ is a point of second order on $A$. Let $\mathscr{A} \rightarrow A_{g}^{\prime}$ be the universal family of such pairs, $e: A_{g}^{\prime} \rightarrow \mathscr{A}$ its identity section. Then the expression (3) defines a canonical invertible section of the sheaf

$$
e^{*}\left(O\left(-\Theta_{\alpha}\right)^{8} \otimes\left(\Omega^{g} \mathscr{A} / A_{g}^{\prime}\right)^{4}\right),
$$

i.e. an isomorphism of this sheaf with $0_{\boldsymbol{A}_{g}^{\prime}}$.

Restricting this isomorphism to $M_{g}^{\prime}$, parametrizing pairs $\left(J, O\left(-\Theta_{\alpha}\right)\right)$, we get finally $\lambda_{1 / 2}^{8} \lambda_{1}^{4}=0_{M^{\prime}}$.

d) End of proof.

Putting together a), b), c), we obtain

$$
\lambda_{i+1}=\lambda_{1}\langle\Omega, \Omega\rangle^{i(i+1) / 2}=\lambda_{1}\left(\lambda_{1}^{12}\right)^{i(i+1) / 2}=\lambda_{1}^{6 i^{2}+6 i+1} .
$$

${ }^{1}$ A similar argument may also be found in [7] 
The independence of this isomorphism of the choice of $\Omega^{1 / 2}$ follows for $g \geqq 3$ from global topological considerations. For a precise statement see the appendix.

\section{Distinguished Bases}

The only advantage of this proof with respect to Mumford's original one (Riemann-Roch-Grothendieck plus global topological arguments) is that it gives rise to explicit formulae. To derive them, we shall now construct distinguished bases in the sheaves $R^{k} \pi_{*} \Omega^{i / 2}$, hence in $\lambda_{i / 2}$, compatible with various canonical identifications. In this subsection we deal with the family $\pi: X \rightarrow M_{g}^{-}$, where $M_{g}^{-}$is a subspace of $M_{g}^{\prime}$, parametrizing pairs (curve, odd theta characteristics $L_{\alpha}$ with $h^{0}\left(L_{\alpha}\right)=1$. The base $M_{g}^{-}$projects onto $M_{g} ; \Omega^{1 / 2}$ is, by definition, $L_{\alpha}$.

a) A basis in $\lambda_{1 / 2}$. The section $v_{\alpha}=\varphi_{0}^{1 / 2}$, defined by (1), Sect. 1, generates $\pi_{*} \Omega^{1 / 2}$. Serre's duality identifies $\left(R^{1} \pi_{*} \Omega^{1 / 2}\right)^{-1}$ with $\pi_{*} \Omega^{1 / 2}$. Hence $\lambda_{1 / 2}=\left(\pi_{*} \Omega^{1 / 2}\right)^{2}$ acquires a generator $d_{1 / 2}=v_{\alpha}^{2}$.

b) A basis in $\lambda_{1}$. Choose a basis $\left(\varphi_{0}, \ldots, \varphi_{g-1}\right)$ in $\pi_{*} \Omega^{1}$ according to (2), Sect. 1 . Identify $R^{1} \pi_{*} \Omega^{1}$ with $0_{M_{g}}$ by the Serre duality. The distinguished generator of $\lambda_{1}$ is $d_{1}=\varphi_{0} \wedge \ldots \wedge \varphi_{g-1}$.

Recall that $B$ is a matrix, transforming $\omega$ into $\varphi$ (Sect. 1, $\mathrm{N}^{\circ} .5$ ).

We assert now that the isomorphism $0_{M_{\bar{g}}}=\lambda_{1 / 2}^{8} \lambda_{1}^{4}$ maps the unit section of $0_{M_{\bar{g}}}$ to

$$
\left(v_{\alpha}^{2}\right)^{8}\left(\omega_{1} \wedge \ldots \wedge \omega_{g}\right)^{4}=d_{1 / 2}^{8} d_{1}^{4}(\operatorname{det} B)^{-4} .
$$

To see this, denote by $p: X \times J_{g-1} \rightarrow J_{g-1}$ the projection, by $L$ the universal sheaf of degree $g-1$ on $X \times J_{g-1}$. Then $d(L)$, constructed with respect to $p$, is canonically isomorphic to $O\left(-\Theta_{g-1}\right)$. In fact, on the complement $J_{g-1} \backslash \Theta_{g-1}$ we have $d(L)=0_{J}$, and the unit section $t$ of this sheaf has a pole of the first order at $\Theta_{g-1}$. At a simple point $\alpha \in \Theta_{g-1}$ this isomorphism is given by the formula $t_{\alpha} \mapsto\left(s s^{\prime}\right)_{\alpha}$, where $s \in H^{0}\left(X, L_{\alpha}\right), s^{\prime} \in H^{1}\left(X, L_{\alpha}\right)^{*}=H^{0}\left(X, \Omega^{1} \otimes L^{-1}\right)$ are such that $(s s)_{\alpha}=(d t)_{\alpha}$. In particular, if $\alpha$ is a theta characteristic, $t_{\alpha}$ goes into $\left(\sqrt{d t_{\alpha}}\right)^{2}$. But this is precisely $v_{\alpha}^{2}$ from (1), Sect. 1.

c) A basis in $\lambda_{3 / 2}$. The sheaf $\pi_{*} \Omega^{3 / 2}$ has the distinguished basis

$$
\left(v_{\alpha} \varphi_{0}, v_{\alpha} \varphi_{1}, \ldots, v_{\alpha} \varphi_{g-1} ; \psi_{1}, \ldots, \psi_{g-2}\right),
$$

where $\psi_{j}$ fulfill the conditions

$$
\psi_{j}=\delta_{j k}\left(d t_{k}\right)^{3 / 2}+O\left(t_{k}\left(d t_{k}\right)^{3 / 2}\right) \text { near } P_{k},
$$

$j, k=1, \ldots, g-2$. Observe that the principal part of $\psi_{j}$ near $P_{g-1}$ is determined from the equation $\sum_{1}^{g-1} \operatorname{res}_{p^{k}}\left(v_{\alpha}^{-1} \psi_{j}\right)=0$. Clearly (5) determines $\psi_{j}$ up to differentials vanishing at all $P_{k}$, but one normalizes $\psi_{j}$ in a unique way, as in (2), Sect. 1, if one demands the vanishing of the term $v_{\alpha}^{3 / 2}=t_{k}^{3}\left(d t_{k}\right)^{3 / 2}$ in the expansion of $\psi_{j}$ near $P_{k}$. The other choices below can be normalized similarly.

The distinguished generator of $\lambda_{3 / 2}$ is

$$
d_{3 / 2}=v_{\alpha} \varphi_{0} \wedge v_{\alpha} \varphi_{1} \wedge \ldots \wedge \psi_{g-2} .
$$

d) A basis in $\lambda_{(i+1) / 2}, i \geqq 3$. Since one has $R^{1} \pi_{*} \Omega^{i / 2}=0$ for $i \geqq 3$, the above game may be continued further inductively. One declares that the distinguished basis in $\pi_{*} \Omega^{(i+1) / 2}$ is one in $\pi_{*} \Omega^{i / 2}$, multiplied by $v_{\alpha}$, plus the differentials with principal parts $\delta_{j k}\left(d t_{k}\right)^{(i+1) / 2}$ at $P_{k}, j, k=1, \ldots, g-1$. 
The distinguished generator of $\lambda_{(i+1) / 2}$ is

$$
\begin{aligned}
d_{(i+1) / 2}= & \text { the wedge product of the elements } \\
& \text { of the distinguished basis of } \pi_{*} \Omega^{(i+1) / 2} .
\end{aligned}
$$

e) A basis in $\left\langle\Omega^{1 / 2}, \Omega^{1 / 2}\right\rangle$. Put $D=\sum_{1}^{g-1} P_{k}$. According to Proposition 5c, we $\underset{g-1}{\text { have }}\left\langle\Omega^{1 / 2}, O(D)\right\rangle=\bigotimes_{1}^{g-1} \Omega_{P_{i}}^{1 / 2}$, and $\left\langle v_{\alpha}, 1\right\rangle$ under this isomorphism maps into $\bigotimes_{1}^{g-1} v_{P_{i}}$, where $v_{P_{\imath}}$ is $v_{\alpha} \bmod t_{i} v$, i.e. $t_{i} d t_{i}^{1 / 2} \bmod t_{i}^{2}$. The isomorphism $O(D) \stackrel{v_{\alpha}}{\longrightarrow} \Omega^{1 / 2}$, $1 \mapsto v_{\alpha}$, sends this class into the distinguished generator of $\left\langle\Omega^{1 / 2}, \Omega^{1 / 2}\right\rangle$, which we denote

$$
\left\langle\mathrm{id}, v_{\alpha}\right\rangle\left(\bigotimes_{1}^{g-1} v_{P_{\imath}}\right)=\prod_{1}^{g-1}\left\langle v_{P_{i}}, v\right\rangle=v .
$$

Now the formal properties of the functors $d$ and $\langle$,$\rangle imply, that$

$$
\begin{aligned}
d_{i+1}= & d_{i} v^{4 i} \text { via identification } \lambda_{i+1}=\lambda_{i}\langle\Omega, \Omega\rangle^{i} \\
& \left(\mathrm{~N}^{\circ} .7 \mathrm{a}\right) \\
d_{1}^{2}= & d_{1 / 2}^{2} v \text { via identification } \lambda_{1}^{2}=\lambda_{1 / 2}^{2}\left\langle\Omega^{1 / 2}, \Omega^{1 / 2}\right\rangle \\
& \left(\mathrm{N}^{\circ} .7 \mathrm{~b}\right)
\end{aligned}
$$

\section{Proof of Theorem 6, Sect. 1}

Putting together (6), (7), and (4), we get formally

$$
d_{2}=d_{1} v^{4}=d_{1}^{9} d_{1 / 2}^{-8}=d_{1}^{13}(\operatorname{det} B)^{-4},
$$

which implies the statement a) of the theorem. To deduce b), note that

$$
\operatorname{det}\left(\frac{i}{2} \int_{X} \omega_{a} \wedge \bar{\omega}_{b}\right)=\operatorname{det} \operatorname{Im} \tau,
$$

hence

$$
\operatorname{det}\left(\frac{i}{2} \int_{X} \varphi_{a} \wedge \bar{\varphi}_{b}\right)=\operatorname{det}|B|^{2} \operatorname{det} \operatorname{Im} \tau
$$

\section{Remarks and Complements}

\section{Admissible Metrics}

The formula for the Polyakov measure, given in [14], was derived by almost the same method as the one above. The difference between two formulae owes partly to a different choice of distinguished bases, but mainly to the fact in [14] the machine of special hermitian metrics on invertible sheaves was systematically used. Since it may have a wider use in various contexts, we add here some hints on the derivation of the formula in [14].

Let $(L,||)$ be an invertible sheaf with hermitian metrics on the complex variety of $M$. Its curvature form is the $C^{\infty}(1,1)$-form $\partial \bar{\partial} \log |s|^{2}$, where $s$ is an arbitrary local 
invertible section of $L$. This form is closed, and its cohomology class equals $2 \pi i c_{1}(L)$. If $M$ is compact, one gets in this way a bijection

$$
\left[\begin{array}{l}
(1,1) \text {-forms } \\
\text { in the class } c_{1}(L)
\end{array}\right] \leftrightarrow\left[\begin{array}{l}
\text { metrics on } L \\
\text { up to proportionality }
\end{array}\right] \text {. }
$$

On a Riemann surface $X$ of genus $g \geqq 1$ there is a canonical $(1,1)$-form

$$
\varrho_{X}=i / 2 g \omega^{t}(\operatorname{Im} \tau)^{-1} \wedge \omega,
$$

where $\omega$ is the Riemann basis. Therefore any invertible sheaf $L$ admits metrics with the curvature form proportional to $\varrho_{X}$. Such metrics are called admissible. For example, the sheaf $O(P)$ has a canonical admissible metric, defined by $\left|t_{P}\right|(Q)$ $=G(P, Q)$, where $G$ is the exponentiated Green function of the Laplace operator $\Delta=-\frac{i}{\pi \varrho_{X}} \partial \bar{\partial}$. To be more precise,

$$
\begin{gathered}
f(P)=-\int_{X} \log G(P, Q) \Delta f(Q) \varrho_{X}(Q) \text { for } \int_{X} f \varrho_{X}=0, \\
\int_{X} \log G(P, Q) \varrho_{X}(Q)=0 .
\end{gathered}
$$

Faltings [9] has shown, that if an admissible metric on a sheaf $L$ is given, then $d(L)$ can be endowed with a canonical metric that transforms in an explicit way under the canonical isomorphisms of Sect.2. One can then use it instead of distinguished bases to compute $\left|\mu_{X}\right|^{2}=d \pi_{g}$ (but not $\mu_{X}$ itself).

For example, the isomorphism

$$
d(L)=d\left(L\left(-\sum P_{i}\right)\right) \otimes\left(\bigotimes_{i} L_{P_{i}}\right),
$$

which we used constantly, becomes an isometry, if $d(L)$ and $d\left(L\left(-\sum P_{i}\right)\right.$ are endowed with Faltings' metrics, while $\otimes L_{P_{i}}$ gets the metrics

$$
\otimes\left(\text { induced metrics on } L_{P_{i}}\right) \otimes \prod_{i<j} G\left(P_{i}, P_{j}\right)^{-1} \text {. }
$$

Such arguments, together with another result of Faltings on the metric change via $\lambda_{1}^{13} \rightarrow \lambda_{2}$ give the formula of [14].

\section{The Problem of Measure for Superstrings}

One hopes that Polyakov's measure for the superstring in the critical dimension 10 should also be computable in the same vein, through an intermediary MumfordBerezin form on a superalgebraic moduli space $M_{g}^{s}$ of dimension $3 g-3 \mid 2 g-2$ (for $g \geqq 2$ ). At the moment, however, only some parts of the whole picture are on a mathematically sound base.

a) It is unknown (at least, to the authors), whether an analogue of the BelavinKnizhnik theorem is true.

b) The supervariety $M_{g}^{s}$, or rather an infinite covering of it, was constructed by one of us (Yu. I. M.) by means of Schottky's superuniformization, but not all techniques needed for transposition of calculations of Sect. 2 are worked out yet. 
At our present level of knowledge, we can calculate at least the analog of the Mumford form on the "component" version of the superspace $M_{g}^{s}$, which parametrizes certain (1|1)-dimensional superalgebraic curves with superconformal structure in the sense of [6], having odd typical structural theta characteristics. By definition, the underlying space of this superspace is $M_{g}^{-}$(see Sect. $2, \mathrm{~N}^{\circ} .8$ ), and the sheaf of holomorphic superfunctions is $\Lambda^{*}\left(\pi_{*} \Omega^{3 / 2}\right)$, where $\pi: X \rightarrow M_{g}^{-}$is the universal family.

Denote this supervariety by $\tilde{M}_{g}^{s}$ and consider the family of supercurves $\tilde{\pi}: \tilde{X} \rightarrow M_{g}^{s}$, where odd superfunctions on $\tilde{X}$ are generated by the functions, lifted from $M_{g}^{s}$, and the sections of $\Omega^{1 / 2}$, lifted from $X$.

On $\tilde{M}_{g}^{s}$ there are analogs of Mumford's sheaves

$$
\Lambda_{i}=\operatorname{Ber} R \tilde{\pi}_{*}\left(\left(\Omega^{s}\right)^{i / 2}\right),
$$

where $\left(\Omega^{s}\right)^{1 / 2}$ comes from $\Omega^{1 / 2}$. The results of Sect. 2 give us a canonical isomorphism on $M_{g}^{-},\left(\lambda_{1} \lambda_{1 / 2}^{-1}\right)^{5}=\lambda_{2} \lambda_{1 / 2}^{-1}$, which after lifting to $M_{g}^{s}$ becomes an isomorphism $\Lambda_{1}^{5}=\Lambda_{3}$.

The section of $\Lambda_{1}^{-5} \Lambda_{3}$, corresponding to 1 , is a component analogue of a "true" Mumford's form. The second formula of [14] gives its modulus squared. The computations of Sect. 2 give an explicit formula for this section itself in terms of distinguished bases.

\section{Distinguished Bases and Canonical Models of Algebraic Curves}

If a curve $X$ of genus $g \geqq 3$ is not hyperelliptic, then the distinguished basis $\left(\varphi_{0}, \ldots, \varphi_{g-1}\right)$ embeds it into a coordinatized projective space $P^{g-1}$.

An advantage of this choice over Petri's one (see [16, pp. 123-135]) is that this embedding is defined only up to finite ambiguity. In fact, to define it, one fixes a typical odd theta characteristic and $g-1$ cubic roots of unity. The formula for $\varphi_{0}$ in terms of the theta function seems to introduce a transcendental element into this construction, but one may well avoid it. E.g. we can leave $\varphi_{0}$ defined up to multiplication by $c$, and $\varphi_{j}, j \geqq 1$, up to multiplication by $c^{-1 / 3}$ [the normalization (2), Sect. 1] is crucial here).

The ideal of equations for $X$ in this embedding is generated for $g \geqq 4$ by quadratic relations between $\varphi_{j}$ 's. (excepting plane quintics and trigonal curves).

The basic quadratic relations can be written in the form

$$
\varphi_{k} \varphi_{l}=\sum_{i=1}^{3 g-3} a_{k l}^{i} w_{i}, \quad k, l=0, \ldots, g-1 .
$$

Here $\left(w_{i}\right)$ is the distinguished basis of quadratic differentials, and coefficients $a_{k l}^{i}$ can be calculated through the expansion coefficients of $\varphi_{i}$ by $v_{\alpha}$ near $P_{k}$.

This refinement of the Petri method may be of independent algebro-geometric interest.

\section{Appendix. Simultaneous Normalization of Mumford's Forms}

In the main body of the paper Mumford's forms $\mu_{g}$ were defined only up to multiplication by a constant, depending on $g$. In this appendix we shall show how 
to normalize $\mu_{g}$ in a canonical coherent way by purely algebraic means. Hopefully, in this normalization $d \pi_{g}=e^{\beta(2-2 g)}\left|\mu_{g}\right|^{2}$ for a certain (zero?) value of $\beta$. This conjecture is equivalent to a factorization property of $d \pi_{g}$.

To achieve this goal, we need to fix the behaviour of $\mu_{g}$ when a curve acquires a singularity. Let us first introduce some notation. Below "curve" means "projective algebraic curve, possibly reducible or even disconnected, with quadratic singularities only." For a curve $X$, let $\Omega^{1} X$ be the sheaf of 1 -forms on it and $\omega_{X}$ the dualizing sheaf (which is always invertible). They are related by a canonical morphism $\left.i: \Omega^{1} X \rightarrow \omega\right)_{X}$, which is an isomorphism off singular points. The image of $i$ coincides with $\pi_{*} \Omega^{1} \tilde{X}$, where $\pi: \tilde{X} \rightarrow X$ is the normalization map, pulling apart the double points. Now let $\alpha$ be such a double point; $x_{1}, x_{2}$ parameters of two branches, crossing at $\alpha$. The one-dimensional fibers of Ker $i$, Coker $i$ at $\alpha$ are generated by $x_{1} d x_{2}, x_{1}^{-1} d x_{1}-x_{2}^{-1} d x_{2}$ respectively. Put

$$
\begin{gathered}
\lambda_{j}(X)=\operatorname{det} R \Gamma\left(X, \omega_{X}^{\otimes j}\right), j \in \mathbb{Z} ; \quad \tilde{\lambda}(X)=\operatorname{det} R \Gamma\left(X, \Omega^{1} X\right), \\
\delta(X)=\lambda_{1}(X) \tilde{\lambda}(X)^{-1} .
\end{gathered}
$$

These are one-dimensional vector spaces with the following properties.

i) They vary in a holomorphic way, when $X$ varies. The symmetries of $X$ act upon them.

ii) There is a canonical element $\operatorname{det} i \in \delta(X)$, vanishing iff $X$ is singular.

iii) (Serre's duality). $\lambda_{j}(X)=\lambda_{1-j}(X)$.

iv) (Disjoint sums). If $X=X_{1} \sqcup X_{2}$, then there are canonical identifications $\lambda_{j}(X)=\lambda_{j}\left(X_{1}\right) \otimes \lambda_{j}\left(X_{2}\right)$, and similarly for $\tilde{\lambda}, \delta$.

For $\lambda_{j}$ this comes from the exact sequence $0 \rightarrow \omega_{1}^{\otimes j} \rightarrow \omega_{X}^{\otimes 1} \rightarrow \omega_{X_{2}}^{\otimes j} \rightarrow 0$; similarly for $\tilde{\lambda}$; and $\delta$ expresses through $\lambda_{1}, \tilde{\lambda}$. Observe that this isomorphism for $\lambda_{j}, \tilde{\lambda}$ multiplies by $(-1)^{\chi\left(0_{X_{1}}\right) \times\left(0_{x_{2}}\right)}$, if one changes the order of $X_{1}, X_{2}$; while one for $\delta$ does not depend on this order.

v) (Glueing points). Let $X$ be a curve, $\alpha_{1}, \alpha_{2}$ smooth points on it. Denote by $\bar{X}$ a curve, obtained from $X$ by glueing these points together into a point $\alpha$. Put $l_{i}=\left(\Omega^{1} X\right)_{\alpha_{i}}$. Then there are canonical identifications

$$
\begin{gathered}
\lambda_{j}(\bar{X})=\lambda_{j}(X)\left(l_{1} l_{2}\right)^{-j(j-1) / 2}, \quad \tilde{\lambda}(\bar{X})=\tilde{\lambda}(X) l_{1} l_{2}, \\
\delta(\bar{X})=\delta(X)\left(l_{1} l_{2}\right)^{-1} .
\end{gathered}
$$

To see this, consider the glueing map $\pi: X \rightarrow \bar{X}$, and let $x_{1}, x_{2}$ be parameters at $\alpha_{1}, \alpha_{2}$. This data defines the following exact sequences:

a)

$$
0 \rightarrow \omega_{X}^{\otimes j} \rightarrow \pi_{*}\left(\omega_{X}\left(\alpha_{1}+\alpha_{2}\right)\right)^{\otimes j} \stackrel{\varphi}{\longrightarrow} \mathbb{C}_{\alpha} \rightarrow 0 .
$$

$$
\begin{gathered}
0 \rightarrow \omega_{X}^{\otimes j} \rightarrow\left(\omega_{X}\left(\alpha_{1}+\alpha_{2}\right)\right)^{\otimes j} \rightarrow 0_{X} / m_{\alpha_{1}}^{j} \oplus 0_{X} / m_{\alpha_{2}}^{j} \rightarrow 0 . \\
0 \rightarrow\left(l_{1} l_{2}\right)_{\alpha} \stackrel{\psi}{\longrightarrow} \Omega^{1} \bar{X} \rightarrow \pi_{*} \Omega^{1} X \rightarrow 0 .
\end{gathered}
$$

Here the maps $\varphi$ and $\psi$ are defined by

$$
\begin{gathered}
\varphi\left(f_{1}\left(x_{1}^{-1} d x_{1}\right)^{j}+f_{2}\left(x_{2}^{-1} d x_{2}\right)^{j}\right)=f_{1}\left(\alpha_{1}\right)+(-1)^{j-1} f_{2}\left(\alpha_{2}\right), \\
\psi\left(c\left(d x_{1}\right)_{\alpha_{1}}\left(d x_{2}\right)_{\alpha_{2}}\right)=c x_{1} d x_{2} .
\end{gathered}
$$


Applying to these exact sequences $\operatorname{det} R \Gamma$, we get:

$$
\begin{gathered}
\lambda_{j}(\bar{X}) \stackrel{(a)}{=} \operatorname{det} R \Gamma\left(X, \omega_{X}\left(\alpha_{1}+\alpha_{2}\right)^{\otimes j}\right) \stackrel{(b)}{=} \lambda_{j}(X) \operatorname{det}\left(0_{X} / m_{\alpha_{1}}^{j} \oplus 0_{X} / m_{\alpha_{2}}^{j}\right) \\
=\lambda_{j}(X) \bigotimes_{a=j}^{0} \operatorname{det}\left(m_{\alpha_{1}}^{a-1} / m_{\alpha_{1}}^{a} \oplus m_{\alpha_{2}}^{a-1} / m_{\alpha_{2}}^{a}\right)=\lambda_{j}(X)\left(l_{1} l_{2}\right)^{j(j-1) / 2}, \\
\tilde{\lambda}(\bar{X}) \stackrel{(c)}{=} \tilde{\lambda}(X) l_{1} l_{2} .
\end{gathered}
$$

The isomorphism for $\delta$ comes from ones for $\lambda_{1}, \tilde{\lambda}$. Again, the isomorphisms for $\lambda_{j}, \tilde{\lambda}$ change sign, when the order of $\alpha_{1}, \alpha_{2}$ is reversed.

vi) (Projective line). If $X$ is a smooth curve of genus zero, then $\lambda_{j}(X)=\tilde{\lambda}(X)$ $=\delta(X)=\mathbb{C}$ canonically.

Indeed, by ii), iii) it suffices to look at the case $\lambda_{j}, j \geqq 0$. Choose a point $x \in X$. Consider an exact sequence

$$
0 \rightarrow \omega_{X}^{\otimes j} \rightarrow \omega_{X}^{\otimes j}((2 j-1) x) \rightarrow F \rightarrow 0,
$$

where $F=\omega_{X}^{\otimes j}((2 j-1) x) / \omega_{X}^{\otimes j}$. The middle sheaf is acyclic, being isomorphic to $0_{X}(-1)$, hence $\lambda_{j}(X)=(\operatorname{det} F)^{-1}$. But $\operatorname{det} F=\mathbb{C}$, under the isomorphism, mapping 1 to $t^{-1}(d t)^{j} \wedge \ldots \wedge t^{-2 j+1}(d t)^{j}$, where $t$ is an arbitrary parameter at $x$. This isomorphism does not depend on choices made to construct it.

Now put

$$
\bar{\lambda}_{j}=\lambda_{j} \delta^{-j(j-1) / 2}, \quad v_{j}=\bar{\lambda}_{j} \bar{\lambda}_{1}^{-6 j^{2}+6 j-1} .
$$

Clearly, the above implies

vii) The properties i), iii), iv), vi) hold for $\bar{\lambda}_{j}, v_{j}$. In the situation v) we have $\bar{\lambda}_{j}(\bar{X})$ $=\bar{\lambda}_{j}(X), v_{j}(\bar{X})=v_{j}(X)$ ("factorization property").

We can state now the main result of this appendix.

Proposition. There is a unique identification $v_{j}(X)=\mathbb{C}$ compatible with $\left.\mathrm{i}\right)$, iv), and $\left.\mathrm{v}\right)$. It is then automatically compatible with iii) and for $X=P^{1}$ coincides with vi). The element $\mu^{(j)}(X) \in v_{j}(X)$ is the normalized Mumford form (or, rather, its value at $X$ ).

Sketch of Proof. Assume first that such $\mu$ exists. According to iv), v) it is defined by its values on smooth connected $X$. But any such $X$ is a member of a family of curves with compact connected parameter space, in which $P^{1}$ with a number of double points also occurs. (Take, e.g. the Deligne-Mumford modular family.) This shows that $\mu\left(P^{1}\right)$, together with i), v), determines $\mu$ uniquely. It remains to see that $\mu\left(P^{1}\right)$ coincides with 1 in normalization vi). Consider a constant family $P^{1} \times A^{1}$ and blow up a point in a fiber over $0 \in A^{1}$. In this new family the fiber over 0 is $P^{1} \sqcup P^{1}$ with two points glued together, i.e. $P^{1} \vee P^{1}$. We have $\mu\left(P^{1} \vee P^{1}\right)=\mu\left(P^{1}\right) \cdot \mu\left(P^{1}\right)$, and $\mu\left(P^{1} \vee P^{1}\right)=\lim _{t \rightarrow 0} \mu\left(P_{t}^{1}\right)$. This forces $\mu\left(P^{1}\right)$ to be 1 .

As for existence of $\mu$, one can prove it by a simple induction on genus, using the structure of Deligne-Mumford moduli space at infinity, together with iv), v) to normalize the Mumford forms. 


\section{References}

1. Polyakov, A.M.: Quantum geometry of bosonic strings. Phys. Lett. 103B, 207-210 (1981)

2. Fradkin, E.S., Tseytlin, A.A.: Quantization of two-dimensional supergravity and critical dimensions in string models. Phys. Lett. 106B, 63-68 (1981)

3. Alvarez, O.: Theory of strings with boundaries: fluctuations, topology and quantum geometry. Nucl. Phys. B 216, 125-184 (1983)

4. Di Vecchia, P., Durhuus, B., Olesen, P., Petersen, J.L.: Fermionic strings with boundary terms. Nucl. Phys. B 207, 77-95 (1982)

5. Baranov, M.A., Svarc, A.S.: On the multiloop contribution in the string theory. Pisma ZETP 42:8, 340-342 (1985)

6. Baranov, M.A., Manin, Yu.I., Frolov, I.V., Svarc, A.S.: Multiloop contribution in fermionic string and Selberg supertrace formula. Yad. Fiz. 39:2, 211-215 (1986)

7. Belavin, A.A., Knizhnik, V.A.: Complex geometry and quantum string theory. Preprint Landau Institute, 32, 1986

8. Mumford, D.: Stability of projective varieties. Enseign. Math. 23, 39-100 (1977)

9. Faltings, G.: Calculus on arithmetic surfaces. Ann. Math. 119:2, 387-424 (1984)

10. Knudsen, F., Mumford, D.: The projectivity of the moduli space of stable curves. I. Preliminaries on "det" and "Div". Math. Scand. 39:1, 19-35 (1976)

11. Deligne, P.: La formule de dualité globale. In: Lecture Notes in Mathematics, Vol.305, pp. 481-587. Berlin, Heidelberg, New York: Springer 1970

12. Mumford, D.: Tata lectures on theta. I, II. Boston: Birkhäuser 1984

13. Mumford, D.: Abelian varieties. Bombay: Oxford UP 1970

14. Manin, Yu.I.: The partition function of the Polyakov string can be expressed through theta function. Pisma ZETP 43:4, 161-163 (1986)

15. Fay, J.D.: Theta function on Riemann surfaces. In: Springer Lecture Notes in Mathematics, Vol. 352. Berlin, Heidelberg, New York: Springer 1975

16. Arbarello, E., Cornalba, M., Griffiths, P.A., Harris, J.: Geometry of algebraic curves, Vol. 1. Berlin, Heidelberg, New York: Springer 1985

17. Bismut, J.-M., Freed, D.S.: The analysis of elliptic families. I, II, Commun. Math. Phys. 106, 159-176 (1986) and to appear

18. Bost, J.B., Jolicoeur, T.: A holomorphy property and critical dimension in string theory from an index theorem. Preprint Saclay PhT/86-28, 1986

19. Manin, Yu.I.: New dimensions in geometry. In: Lecture Notes in Mathematics, Vol.1111, pp. 59-101. Berlin, Heidelberg, New York: 1985

20. Ray, D., Singer, I.M.: Analytic torsion for complex manifolds. Ann. Math. 98, 154-180 (1973)

21. D'Hoker, E., Phong, D.H.: On determinants of Laplacians on Riemann surfaces. Commun. Math. Phys. 104, 537-545 (1986)

Communicated by A. Jaffe

Received July 28, 1986

Note added in proof. Since this paper was written we became aware of a very interesting letter by P. Deligne to D. Quillen, dated back to 20 June 1985, where a refinement of the GrothendieckRiemann-Roch for $c_{1}$ of direct images of rank 0 virtual vector bundles over Riemann surfaces was given and the normalized Mumford forms were defined. 